\title{
Cortisol production and the metabolic syndrome
}

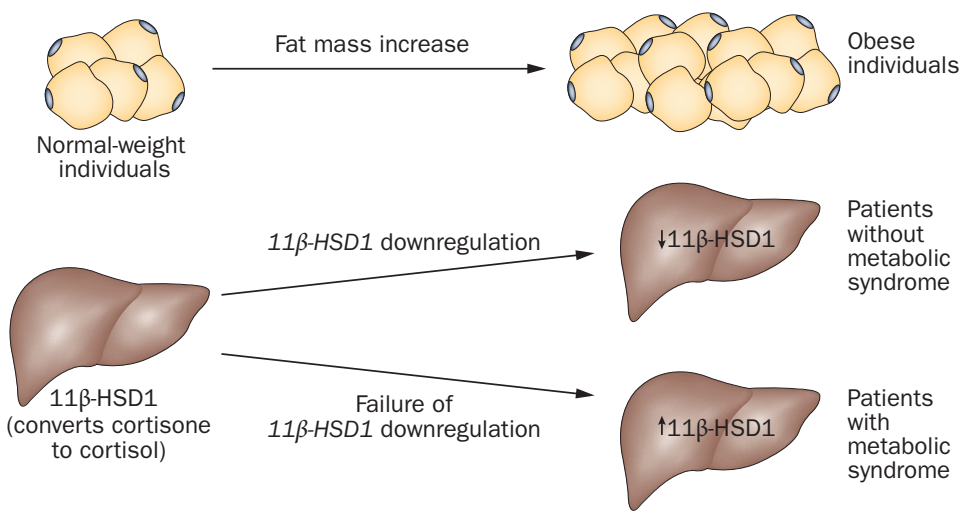

Image created in consultation with G. Fernández-Vázquez.

The liver-specific upregulation of genes involved in cortisol production may have a role in the pathogenesis of the metabolic syndrome, report Gumersindo Fernández-Vázquez and colleagues.

"Our interest in the field dates back to 4 years ago, and was inspired by seminal research performed by several groups," explains Fernández-Vázquez. "This body of knowledge points to a relevant role of local cortisol in the pathogenesis of obesity-related metabolic syndrome." $11 \beta$-hydroxysteroid dehydrogenase type 1 (11 $\beta$-HSD1), which is mainly found in the liver, has a key role because it converts cortisone (inactive) to cortisol.

"In vivo data suggest that hepatic $11 \beta$ HSD1 activity is lower in obese than lean individuals, and that this downregulation fails in those obese patients who have metabolic alterations," says FernándezVázquez. As direct data were lacking, the researchers decided to see if different expression levels of genes involved in cortisol production in the liver and/or fat tissues explained susceptibility to the metabolic syndrome in obese patients.

The study included 50 morbidly obese patients who were undergoing bariatric surgery. Of these patients, 20 were classified as having metabolic syndrome and the other
30 were classified as not having metabolic syndrome. Liver and subcutaneous and visceral adipose tissue biopsy samples were obtained at the time of bariatric surgery. Gene expression levels were measured by real-time quantitative PCR.

The results showed that the livers of obese patients with metabolic syndrome had higher expression levels of $11 \beta-H S D 1$ than the livers of obese patients without metabolic syndrome.

"These results broaden the current knowledge in the field and support in vivo data indicating that obese patients with metabolic syndrome have higher levels of cortisol production-which may contribute to metabolic derangements," says Fernández-Vázquez.

Future work is likely to focus on two main issues: first, what is the cause and effect relationship between increased cortisol production and the metabolic syndrome; and second, how does the $11 \beta$-HSD1 gene become dysregulated?

Isobel Franks

Original article Torrecilla, E. et al. Liver upregulation of genes involved in cortisol production and action is associated with metabolic syndrome in morbidly obese patients. Obes. Surg. doi:10.1007/s11695-011-0524-9 